\title{
Microelectrónica y educación especial en el Reino Unido
}

\author{
Julio González
}

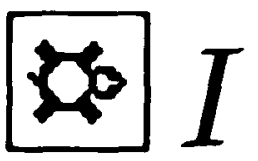

El Reino Unido ha sido uno de los países pioneros en la utilización de los ordenadores y la electrónica para mediar y prolongar los procesos psicológicos de las personas con déficit $y$ contribuir asi a su desarrollo y normalización funcional. Un adecuado conocimiento del estado de la cuestión en ese país puede, además de facilitar una información factual de los grandes avances producidos en comunicación y control del entorno, servir como modelo o banco de sugerencias para la acción en nuestro país.

\section{INGLATERRA, PAIS DE GALES E IRLANDA DEL NORTE}

En el momento actual el desarrollo de las aplicaciones de la microelectrónica a sujetos con necesidades educativas especiales depende de la $\mathrm{Mi}$ croelectronics Education Support Unit (MESU), fundada en marzo de 1986 por el Department of Education and Science para un periodo de cinco años. Se trata, en realidad, del sucesor del anterior Microelectronics Education Programme (MEP) lanzado originariamente por el gobierno británico en el año 1980 y cuya actividad fue desplegada a lo largo de los siguientes seis años. MESU, con un presupuesto anual de tres millones de libras esterlinas, abarca a la población escolar en Inglaterra, País de Gales e Irlanda del Norte; como después se detalla, Escocia se rige por su propio programa independiente.

La MESU se propone como objetivo fundamental, a medio plazo, el establecer firmemente la utilización de soporte microelectrónico por parte del personal docente que incide sobre niños con distintas minusvalías o discapacidades.

La Dirección General de la Unidad se sitúa en Coventry, en la Universidad de Warwick, donde el Director (actualmente John Foster) tiene su Equipo Central (Central Team). De aquí dependen cuatro SEMERCs (Special Education Microelectronic Resource Centres), un $A C E$ (Aids for Communication in Education) Centre y un Special Needs Software Support Centre. 


\section{Los SEMERCs}

Los Special Education Microelectronic Resource Centres (SEMERC) son centros de recursos que, con un amplio rango de software y hardware, actúan como foco de actividad y asesoramiento para una zona determinada.

Existen cuatro centros ubicados en Bristol, Manchester, Newcastle y Redbridge, respectivamente; cubriendo cada uno de ellos un ámbito territorial de aproximadamente unas 25 Local Education Authorities (LEA) que, como es sabido, constituyen las unidades administrativas básicas del descentralizado modelo educativo inglés. En cada LEA el SEMERC cuenta con uno o varios coordinadores a través de los cuales el centro desarrolla su labor hacia las escuelas.

Entre sus actividades destaca la distribución del «Blue File»Software, o programas informáticos susceptibles de copia libre. El SEMERC envía el software a su contacto personal del LEA quien a su vez se encarga de hacerlo llegar a los centros escolares que previamente lo habían solicitado. En todo momento se disponen de listas actualizadas del software existente junto a las principales características de cada programa.

Otro importante campo de actividad es el que se refiere a la ayuda práctica a los profesores de niños con necesidades especiales, principalmente en: nicos.

- Información y asesoramiento sobre el uso de equipos microelectró-

- Entrenamiento para casos puntuales.

- Demostraciones generales del software y hardware disponible.

- Constituirse en centros de discusión y lugar de encuentro de profesores con alumnos discapacitados.

Al mismo tiempo, los SEMERCs trabajan directamente sobre un reducido número de centros escolares que a modo de centros piloto («exemplars» o «hot spots») representan el marco en el que se llevan a cabo determinadas aplicaciones con carácter experimental.

\section{El ACE Centre}

El ACE (Aids for Communication in Education) Centre comenzó a funcionar en mayo de 1984. Ubicado en la Ormerod School de Oxford, constituye un foco de información y experiencia en el uso de equipos microelectrónicos para niños con trastornos de comunicación (sintetizadores de voz, teclados especiales, conmutadores, ordenadores portátiles, etc.).

Entre sus funciones sobresalen:

- Recoger y difundir información sobre las ayudas tecnológicas a la comunicación.

- Proporcionar facilidades, asesoramiento y equipos a prueba para casos concretos (niños acompañados por sus padres, profesores o terapeutas).

- Inspección y evaluación de software y equipos especiales.

- Desarrollo de proyectos.

Un miembro del equipo del ACE Centre se halla incorporado al SEMERC de Manchester para coordinar la actuación en el norte del país. 


\section{Special Needs Software Centre}

Este organismo tiene una responsabilidad nacional en la producción y modificación de software para ayuda de niños con necesidades educativas específicas. No obstante, su dotación de recursos humanos se ve limitada a dos programadores y un coordinador experto en educación, por lo que su actuación se somete a fuertes criterios de priorización. Su principal trabajo se centra en adaptar productos ya existentes a las características de los usuarios a que se destinan, canalizándose la distribución a través del sistema «Blue File» ya comentado.

\section{ESCOCIA}

Escocia se rige por un plan propio distinto del llevado a cabo por MESU, y dirigido desde Glasgow por el Scottish Council for Educational Technology.

\section{CALL Centre de Edimburgo}

En este contexto, la institución con mayor presencia en territorio escocés es el Communication Aids for Language and Learning (CALL) Centre, situado en el Departamento de Educación de la Universidad de Edimburgo. Fue fundado unos seis años atrás por varias instituciones y organismos, entre ellos el Scottish Education Department.

De composición multidisciplinar, el equipo se integra básicamente por:

- un psicólogo,

- un logopeda,

- un ingeniero electrónico,

- un instructor de Logo,

- un experto en ayudas a la comunicación,

- un servicio de información,

- un administrador,

- un secretario.

En adición a este staff permanente se incluyen colaboradores temporales (trabajadores sociales, técnicos y programadores, profesores y otros) distribuidos en diferentes puntos geográficos.

La actividad se dirige fundamentalmente hacia el:

- asesoramiento en elección de equipos de ayuda a la comunicación,

- entrenamiento en la utilización de los equipos una vez escogidos,

- desarrollo de nuevos sistemas y dispositivos,

- investigación en aspectos concernientes a las dificultades de comunicación y tecnología,

- demostraciones generales prácticas de empleo de los sistemas.

Periódicamente el CALL Centre organiza seminarios, cursos y encuentros entre los profesionales del campo así como la posibilidad de prestar equipos a prueba por períodos mensuales a instituciones educativas escocesas. 
Cuenta asimismo con un servicio de publicaciones técnicas especializadas en temas monográficos concretos y hojas de información general.

A continuación pasamos a describir brevemente los principales elementos - tanto a nivel de hardware como de software - que configuran el abanico de los recursos materiales más empleados.

\section{HARDWARE}

\section{Ordenadores}

El equipo más extendido es el $B B C$, que viene a ser el ordenador «oficial» en Gran Bretaña, tanto en la educación ordinaria como en la especial. En España se está utilizando principalmente en el País Vasco —el centro ASPACE de Guipúzcoa, en colaboración con la Facultad de Informática de San Sebastián y el programa IMI, lleva a cabo una experiencia con paralíticos cerebrales tomando como modelo las aportaciones del Reino Unido- Especialmente diseñado para la educación y con un extenso repertorio de programas y periféricos tiene, no obstante, el inconveniente de su falta de comercialización fuera de aquel país. Los modelos más empleados son el BBC B, el BBC B+ y el BBC Master 128.

Otro sistema de gran implantación es el $R M L$ (Research Machines, Ltd.) con los modelos $480 \mathrm{Z}$ y Nimbus.

Pese a todo, se observa una creciente preocupación por adaptar el software hacia modelos IBM PC y Compatibles, actualmente en expansión. No en vano el Council for Educational Technology ha promovido la iniciativa de caminar hacia un acuerdo que permita desarrollar normas de compatibilidad unificadas para ayudas a la comunicación en las comunidades europeas. Esto ya ha dado lugar a varias reuniones de representantes de los distintos paises de la CEE, entre los que se incluye España.

\section{Dispositivos periféricos de entrada}

Con frecuencia los sistemas estándar no permiten un manejo adecuado por parte de los sujetos con discapacidades, por lo que se ha de recurrir a dispositivos que faciliten la comunicación con la máquina, tanto en lo que se refiere a la entrada (INPUT) como a la salida (OUTPUT) de información.

El muestrario es variado:

\section{Conmutadores (Switches)}

Son los mecanismos más sencillos y a la vez más diversos. Especialmente indicados en el terreno de las deficiencias motóricas, de manera que aprovechando cualquier movimiento, por pequeño y residual que sea, posibiliten la interacción con el equipo. También son necesarios en el campo de la deficiencia mental para simplificar las opciones de entrada y dar lugar a un manejo más sencillo. 
En esencia consisten en cualquier dispositivo que cierre un circuito eléctrico durante el tiempo en que sea accionado. Se produce así una señal que es detectada e interpretada por el ordenador. Las posibilidades son múltiples y adaptables a las características particulares de cada persona:

- De presión: con distintos formatos y posibilidades en cuanto a número, tamaño y movilidad (pedales, conmutadores manuales, para movimientos de cuello, antebrazos, etc.). Ejemplos: QED Pedal Switch; Platform Switch.

- Por succión o soplo: para aquellos sujetos imposibilitados de ejecutar cualquier movimiento con la cabeza o los miembros. El conmutador reside en el interior de un tubo provisto de boquilla y se acciona mediante succión o soplo. Ejemplo: los del Magpie System.

- Por orientación: los cambios de orientación en el espacio producen una señal merced a una bola interna de metal sólido o de mercurio; se acoplan a cualquier parte del cuerpo. Ejemplo: Mercury Sivitch.

- Por sonido: claves acústicas que cambian de estado ante la emisión de cualquier sonido que sobrepase un umbral determinado. Ejemplos: Hand-beld Micromike; Desk-top Micromike (ambos incluidos en el Magpie System).

- Por luz: se activan al interceptar un haz de luz o bien desde dispositivos acoplados a la cabeza provistos de una fuente luminosa que se proyecta sobre conmutadores fotosensibles. Ejemplos: Photonic Wand.

\section{Teclados especiales}

Sustituyen a los convencionales (tipo QWERTY) con distintos fines.

- Expandidos: muchos más grandes que el estándar e indicados para sujetos de pobre precisión motriz. Ejemplos: Expanded BBC Keyboard; o el Delves Keyboard, formado por ocho grandes teclas en línea.

- Reducidos: de pequeñas dimensiones y diseñados para personas con bajo tono muscular y escasa capacidad de desplazamiento. Ejemplos: Mini Keyboard; o el POP-IT, el más pequeño, con unas dimensiones de $4 \times 4$ pulgadas.

- Carcasas (Keyguards): estructuras perforadas que se superponen a los teclados normales para facilitar la pulsación de las teclas. Ejemplos: Keyguard $B B C B$ y $B B C$ Master 128.

- Simuladores de teclado (Keyboard Emulator): permiten el control de los programas estándar a través de una segunda pantalla en la que se presenta una copia del teclado del ordenador y donde cada tecla puede ser seleccionada a partir de únicamente 1 ó 2 conmutadores. Este modo de selección se denomina "sistema de barrido" y se trata de que un cursor -0 cualquier otro sistema de señalización como cambio de color, sombreado, etc.- se va desplazando en la pantalla de tecla en tecla a cada señal enviada por el sujeto y, una vez que se llega a la deseada, ésta es seleccionada con una segunda señal. Aunque el procedimiento es lento, existen modelos acompañados de programas que optimizan las secuencias de barrido según la frecuencia de uso de las distintas teclas. Ejemplos: Keymaster; BBC Keyboard Emulator. 
Teclados de Conceptos (Concept Keyboard)

Los consideramos aparte dada su relevancia y las posibilidades que ofrecen.

A grandes rasgos consisten en una superficie plana, generalmente de policarbonato, montada sobre una estructura de aluminio y dividida en un número variable de celdillas - 128 ó 256 - sensibles al tacto. Cada una de estas celdillas o grupos de ellas pueden ser asignadas por el usuario a una forma, palabra, frase, parte de una escena, etc., que tengamos representadas previamente en una lámina plástica (overlay) al efecto y que se superpone sobre aquél. Cuando el sujeto toca la lámina, el ordenador actúa según las celdillas excitadas en particular. Existen programas abiertos en los que el profesor puede diseñar sus láminas y definir los mensajes o acciones asociadas a cada celdilla.

Es claro que un sistema así proporciona un sinnúmero de aplicaciones dirigidas a una extensa variedad de alumnos en cuanto a edades y características, tanto en la educación especial como en la ordinaria (primaria y secundaria).

Los dos tamaños más populares en Gran Bretaña son el $A 3 / 128$ y el $A 4 / 128$ y se suministran paquetes de programas muy diversos operativos en los ordenadores anteriormente reseñados. Además interfaces apropiadas hacen posible su utilización con otros modelos como el Spectrum, Apple IIe, Commodore, IBM y Compatibles, etc.

Las casi ilimitadas posibilidades se concretan a través de las láminas - y sus programas asociados-, gracias a los cuales este material resulta útil tanto para la discriminación entre dos formas básicas, empleo del sistema BLISS, como para el diseño de un circuito eléctrico o el estudio de una escena cotidiana de la Edad Media. Prueba de la importancia de las mismas es la existencia de publicaciones especializadas en ellas, como es el caso de "OVERLAY", revista publicada a partir de enero de 1988 por la AB European Marketing's Concept Keyboard que, aunque con fines en parte comerciales, da noticias e informaciones sobre material disponible e ideas a desarrollar por los propios usuarios, en apartados como: E. Primaria; Necesidades Especiales; E. Secundaria; Industrial/Comercial y Software.

En la ilustración 2 presentamos un ejemplo de utilización «ad hoc» del teclado de conceptos al caso concreto de una niña galesa con dificultades de lenguaje. La lámina que se reproduce se basa en palabras de su propio vocabulario básico extraídas a partir de la construcción de una historia.

Pantallas de Contacto (Touch Screen): A través de ellas la pantalla del monitor se torna sensible al tacto. Muy indicadas para el desarrollo de la coordinación óculo-motriz. Ejemplo: Touchtech 501 para monitor de 14 pulgadas.

Lápiz óptico (Lightpen): Hacen posible el dibujar o colorear directamente sobre la pantalla o elegir opciones de un menú. Ejemplo: Robin Lightpen.

Joysticks: Son los mandos manuales comúnmente utilizados en los juegos de «arcade», pero constituyen una ayuda en la interacción con el microordenador para algunos niños con dificultades motrices. Existen dos tipos básicos: el «análogo» en el que la señal de salida es proporcional a la 
cantidad de movimiento suministrado, y el común, del tipo de todo o nada, con las cuatro direcciones espaciales principales.

Ratón (Mouse): Periférico estándar de extendido uso en el campo profesional pero igualmente útil en la $\mathrm{E}$. Especial.

Roller balls: Similar al anterior, se compone de unas bolas encastradas en una armadura y que pueden rodar en todas las direcciones. El movimiento de las mismas queda registrado y se reproduce en la pantalla del monitor. Ejemplos: Marconi Tracker Ball; Trackball.

\section{Dispositivos periféricos de salida}

Reciben información de salida desde el ordenador y la traducen en acciones concretas. Gracias a ellos el sujeto se puede comunicar e interactuar con su entorno en un grado que no le sería posible por sus propios medios.

Sintetizadores de voz: equipos especiales capaces de producir voz artificial a partir de un mensaje previo, procedente bien de forma directa desde el sujeto o con la mediación de un microordenador. Muy útiles como medio de comunicación para personas sin habla o como salida de información desde el computador para invidentes o sujetos sin lecto-escritura. Ejemplos: Votrax Type E Talk, para ordenadores RML; Mimic; Superior Speech, para BBC.

Control del entorno: A través del ordenador y los periféricos oportunos, el sujeto puede llevar a cabo acciones sobre su entorno próximo que, de otro modo les resultarían imposibles o muy difíciles. Desde conectar y desconectar interruptores de la red eléctrica, poner en marcha un electrodoméstico, accionar un portero automático, etc., hasta manejar una silla de ruedas con los movimientos de la cabeza.

"Robots": Brazos articulados accionables por personas con dificultades severas a nivel motor; o incluso las «Logo Turtles» (tortugas del lenguaje informático LOGO), vehículos con movimiento propio controlados desde el microordenador y que reproducen fielmente sobre el suelo las acciones ordenadas a partir de instrucciones directas o programas informáticos escritos en ese lenguaje. Ejemplos: Jessop Turtle; Valiant Turtle.

Visispeech: para utilización por parte de los logopedas, presenta visualmente los patrones relevantes del habla constituyendo un modo alternativo de retroalimentación para los sujetos sin aferencia auditiva (o seriamente dañada).

Juguetes adaptados (Adapted Toys): Juguetes a los que se les han practicado las oportunas modificaciones para poder ser controlados desde un conmutador u otro dispositivo. El Sequal Toy Educational Service ofrece un servicio postal de información y asesoramiento sobre el tema. Ejemplos: el Instant Toy Adapter del Magpie System; Clatterway Toys, con un catálogo de 25 juguetes adaptados; Toyaids Project, etc.

\section{Software}

La mayor parte de los programas disponibles están diseñados para rodar en ordenadores $B B C$ y $R M L$, pero actualmente se tienen versiones de muchos de ellos para IBM y Compatibles. De este trabajo de adaptación se encarga principalmente el Computer Centre for the Disabled, Polytechnic of Central London. 
Se han de señalar los Blue File Programs que, como ya hemos adelantado, constituyen un amplio repertorio de programas informáticos desprovistos de cualquier sistema técnico de protección y, por tanto, resultan libremente copiables. Este software procede de dos fuentes principales: en primer lugar, el que se crea institucionalmente en el contexto de MESU a través del Special Needs Software Centre, ya visto, y en segundo lugar a partir de donaciones hechas por profesores que han desarrollado programas propios útiles para su trabajo, los cuales, aunque se les reconoce su propiedad intelectual y el «copyright» sobre los mismos, dejan constancia de su permiso para ser reproducidos con fines exclusivamente educativos en el ámbito del Reino Unido.

Este software se suministra gratuitamente por medio de los SEMERCs en discos de 5.25 pulgadas y 40 pistas en formato DFS (disc filing system). Basta con que el centro o profesional interesado proporcione los discos vírgenes necesarios con la especificación de los programas que se requieren para que, acto seguido, se les devuelva con una copia de los mismos.

Paralelamente se encuentra la red de casas comerciales (ver Apendice) especializadas en software educativo (general y especial), que se rigen por la legislación vigente en el país.

En líneas generales el software disponible puede ser clasificado en las siguientes categorías:

- Language Development and Early Reading (Desarrollo del lenguaje y lectura temprana). Programas diferenciados en dos apartados:

- Non Text Programs. Sin utilización de texto, para sujetos no lectores. Ej.: BLOB; CARTOON; COMPACT; GOING PLACES; PICTURE PLAY.

- Text-Based Programs. Con empleo de un lenguaje escrito sencillo. Ej.: ALL ABOUT ME; CATCHUP; WORDWERB; INTRO TRAY; WORDSEARCHER; MOVING IN.

- Developing Writing Skills (Desarrollo de destrezas grafoescritoras). Con tres niveles progresivos:

- For Beginning Writers. Para principiantes en escritura. Ej.: EASYTYPE; FOLIO; PROMPT/WRITER; STORY; WRITE; WRITER; PROMPT-3.

- For Established Writers. Para sujetos que ya han alcanzado la escritura. Ej.: EDSPELL; INTERWORD; PENDOWN; TRACKS; WORDWISE PLUS.

- Advanced Applications. Ej.: AMX PAGEMAKER; FLEET STREET EDITOR.

- Information Handling (Manejo de Información). Son en realidad diversas bases de datos con distintos niveles de complejidad y dirigidas al campo educativo:

- Classification Programs. Iniciación a tareas sencillas de clasificación. Ej.: THE SORTING GAME; TREE OF KNOWLEDGE.

- Introductory Databases. Ej.: JUNIOR FIND; LIST EXPLORER; MALLORY; PICFILE CATEBY MANOR.

- More Advanced Databases. Ej.: FIND; GRASS; INFORM; SCAN. 
- Life Skill (Habilidades para la vida). Se trata de programas encaminados a desarrollar conductas y destrezas básicas de adaptación a situaciones corrientes de la vida. Suelen simular situaciones reales en los que el sujeto debe tomar decisiones y comprobar las consecuencias de las mismas. El repertorio es amplio: manejo del teléfono, comida, conciencia del peligro, comprar, realizar un menú, manejo de situaciones domésticas, etc. Ej.: AIRBORNE; BANK; CAMPING; EATING FOR HEALTH; GAS METER AND BILLS; HOME; PHONIN; SAFETY; TOWN; SHOPS-SUPERMARKET.

- Logo/Turtle Graphics. Introducción al lenguaje LOGO y manejo de gráficos por tortuga. Se dividen en:

- Pre-Logo Programs. Ej.: COME AND GO; NUDGE; TRUNDLE.

- Turtle Graphics Packages. Ej.: ARROW; DART; LEICESTER TURTLE; OKLOGO.

- Logos. Ej.: LOGOTRON LOGO; LSL LOGO; RM LOGO; ACORNSOFT LOGO.

- General Framework Programs. De propósito general para el manejo sencillo del microordenador. Ej.: BARCHART; CRACK IT; DATAVIEW; TOUCH EXPLORER; TRAY2; TRAY3; WORDPLAY; READMATICS.

- Music. De iniciación musical. Ej.: DRAGON WORLD; MICROPITCH; THE MUSIC SYSTEM; PIEMAN; THE SYNTH.

- Art E Design. Dirigidos hacia tareas plásticas. Se clasifican en tres niveles:

- Simple Drawing and Painting Programs. Ej.: HARLEQUIN; PAINTBRUSH; RAINBOW; SHAPE HAPPY.

- Advanced Art/design Programs. Ej.: CATOON; MOSAIC; PICTURE BUILDER; PAZAZZ.

- Sophisticated Graphics Packages. Ej.: AḾX SUPER-ART; BISTICK 1; IMAGE.

- Mathematics. Existe un amplio repertorio con niveles de dificultad muy distintos y clasificable en diferentes áreas:

- Early Number Skills. Iniciación a los números. Ej.: COUNTING; NUMBER TOUCH; TALK ABOUT NUMBERS.

- Place Value. Ej.: ABACUS; SIZE GAME; ZOOM.

- Addition \& Substraction. Ej.: ADDING \& SUBSTRACTING; MICRO SMILE; PLAY TRAIN; SUMS.

- Multiplication and Division. Ej.: TABLE ADVENTURES.

- Money. Manejo del dinero. Ej.: COINS; TEASHOP; TOYSHOP.

- Shape. Formas básicas. Ej.: SHAPE FINDER; SHAPE SNAP; WEST MIDLANDS PROGRAMS.

- Time. Tiempo. Ej.: CLOCKS; TIMEMAN $1+2$.

- Volume and Mass. Ej.: JARS.

- Measurement. Medidas. Ej.: LEICESTER PROGRAMS. 
- Sequencing and Logic. Con el propósito de desarrollar el razonamiento lógico-matemático. Ej.: LOGICAL ALL SORT; MATHEMATICAL INVESTIGATIONS IN THE CLASSROOM.

\section{Anexo}

\section{DIRECCIONES DE INTERES}

\section{MESU}

Information Officer, Microelectronics Education Support Unit, Advanced Technology Building, Science Park, University of Warwick, Coventry CV4YAL. (0203 416994)

SEMERCs

Bristol SEMERC, Bristol Polytechnic, Redland Hill, Bristol B56 6UZ. (0272 733141)

Manchester SEMERC, Manchester Polytechnic, Hathersage Rd, Manchester M13 OJA. (061 $2259054 \times 289)$

Newcastle SEMERC, Newcastle Polytechnic, Coach Lane Campus, Newcastle upon Tyne NE7 7XA. (091 266 5057)

Redbridge SEMERC, Dane Centre, Melbourne Rd, Ilford, Essex. (01 478 6363)

ACE CENTRE, Ormerod School, Waynflete Rd, Oxford OX3 8DD. (0865 63508)

CALL CENTRE, University of Edinburgh, 4 Buccleuch Place, Edinburgh EH 89 LW

SPECIAL NEEDS SOFTWARE CENTRE, Manchester Polytechnic, Hathersage Rd, Manchester M13 OJA. (061 $2259054 \times 284$ )

COUNCIL FOR EDUCATIONAL TECHNOLOGY, 3 Devonshire St., London W1N 2BA. (01 6364186 )

BRITISH INSTITUTE FOR MENTAL HANDICAP, Wolverhampton Rd, Kidderminster, Worcester DY103PP. (0562 850251)

SCOTTISH COUNCIL FOR EDUCATIONAL TECHNOLOGY, Dowanhill, 74 Victoria Crescent Rd, Glasgow G129JN

IT AND FE STUDENTS WITH MODERATE LEARNING DIFFICULTIES, National Co-ordinator, Peter Fowler, 285 Buxton Rd, Macclesfield, Cheshire SK11 7ET (0625 20051)

COMPATIBILITY GUIDELINES FOR COMMUNICATION AIDS, National Co-ordinator, Lyndon Thomas, Special Needs Technology Centre, 101 Box Lane, Wrexham, Clwyd. (0978 264408)

16 BIT MACHINES FOR SPECIAL EDUCATIONAL NEEDS, Project Manager, Tom Holloway, CCD, Polytechnic of Central London, 115 New Cavendish Street, London W1M 8JS (01-631-3332)

2. CASAS Y CENTROS PROVEEDORES DE SOFTWARE/HARDWARE EDUCATIVO EN EL REINO UNIDO

AB Euro Marketing, Wharfedale Rd, Pentwyn, Cardiff, South Glamorgan CF2 7HB. (0222-733485)

E. J. Arnold, Parkside Lane, Dewsbury Rd, Leeds LS11 5TD. (0532 - 772112)

ASK, Duke St, Wisbech, Cambs PE13 2AE (0945 - 63441) 
BIME, St Martin's Hospital, Wellsway, Bath BA2 5RP

BIMH (British Institute for Mental Handicap), Wolverhampton Rd, Kidderminster, Worcs DY 10 3PP. (0526 - 850251)

Blue File (MESU), These programs may be freely copied for non-profit making educational purposes from LEA Computer Centres, Special Education/Microelectronics Support Teachers, and SEMERCs. Their copyright is held by CET. They can also be purchased at low cost from BIMH (see above).

Bradford Activity Toys, 103 Dockfield Rd, Shipley, Bradford, W Yorks BD17 2AR. $(0274-596030)$

CALL Centre, Edinburgh University, 4 Buccleuth Place, Edinburgh EH8 9TL. (031 667 1011)

Clare's, 98 Middlewich Rd, Rudheath, Northwich, Cheshire CW9 7DA. (0606 48511)

Clatterway Toys, 31 Watton Rd, Colney, Norwich. (0603 - 810471)

Compaid Trust, Unit CH1, Pembury Hospital, Tunbridge Wells, Kent TN2 40J

Computer Aids, (George Derby), The Fox Covert, Fox Covert Lane, Picton Gorse, Chester (0244 300363)

Daco Software, 59 Mackenzie Rd, Moseley, Birmingham B11 4EP (021 449 - 2253)

Delves School, Hayes Lane, Swanwick, Derbys. (0773 - 602198)

Educational Software Co, 108 Parthenon Drive, Liverpool L11 $7 A Q$. (051 226 - 1214)

ESM, Duke St., Wisbech, Cambridgeshire, PE13 2AE. (0945 - 63441)

HPRU (Handicapped Persons Research Unit), Newcastle Polytechnic, Coach Lane Campus, Newcastle upon Tyne NE7 TTW. (091 2358211)

Interface Designs, 12 East Meads, Onslow Village, Guildford, Surrey GU2 5SP. (0483 - 32909)

Jessop-Ralph Ltd, Unit 5, 7 Long St., London E2 8NH. (01 739 - 3232)

Kinder School, Bassetlaw DG Hospital, Worksop, Notts S81 OBD

Liberator Electronic Aids Ltd, 12 Devonshire Close, Amersham, Bucks HP6 5JG. (02403 - 6332)

Magpie Systems, (John Tabberer), 45 Runcorn Rd, Moore, nr Warrington. (0925 - 74704)

MARDIS (Microprocessor Assistance for the Disabled), Room B22, Engineering Building, University of Lancaster, Lancaster LA1 4YW. (0542 - 65201 × 4993)

Micrex, 7 Laverock Lea, Portchester, Hants PO16 8DA. (0705 - 374036)

Microvitec, Futures Way, Bolling Rd, Bradford, W Yorks BD4 7TU. (0274 - 390011)

Mirrorsoft, Maxwell House, 74 Worship St, London EC2

NORDIS Software, Cornhill Close, Lodge Industrial Estate, Harlestone Rd, Northampton NN5 7UB. (0604 54358/9)

Nixon, Alan, 5 Perry Rd, Bangor, Co. Down, N Ireland BT19 2AU

Pyramid Micros, Cairo St, Warrington, Lancs. (0925 - 35713)

RCEVH (Research Centre for the Education of the Visually Handicapped), Selly Wick House, 59 Selly Wick Rd, Birmingham B29 7JE. (021 471-1303)

Spastics Society, 16 Fitzroy Square, London W1P 5HQ. (01 387 - 9571)

Special Acces Systems, 4 Benson Place, Oxford. (0865 - 56154)

STEPS (Sequal Toy Educational Postal Service), Block 178, Milton Trading Estate, Abingdon, Oxon OX14 4ES. (0235 - 833193)

Switch Controlled Software, 116 Stockingston Rd, Luton, Beds LU2 7NH.

Toyaids Project, Lodbourne Farmhouse, Lodbourne Green, Gillingham, Dorset.

Toys for the Handicapped, 76 Barracks Rd, Sandy Land Industrial State, Stourport-on-Severn, Worcestershire DY13 9QB. (02993 - 78820)

Widgit Software Ltd, 1 The Ryde, Hatfied, Herts AL9 5DQ. (070 72 - 64780) 
68

Figura 1

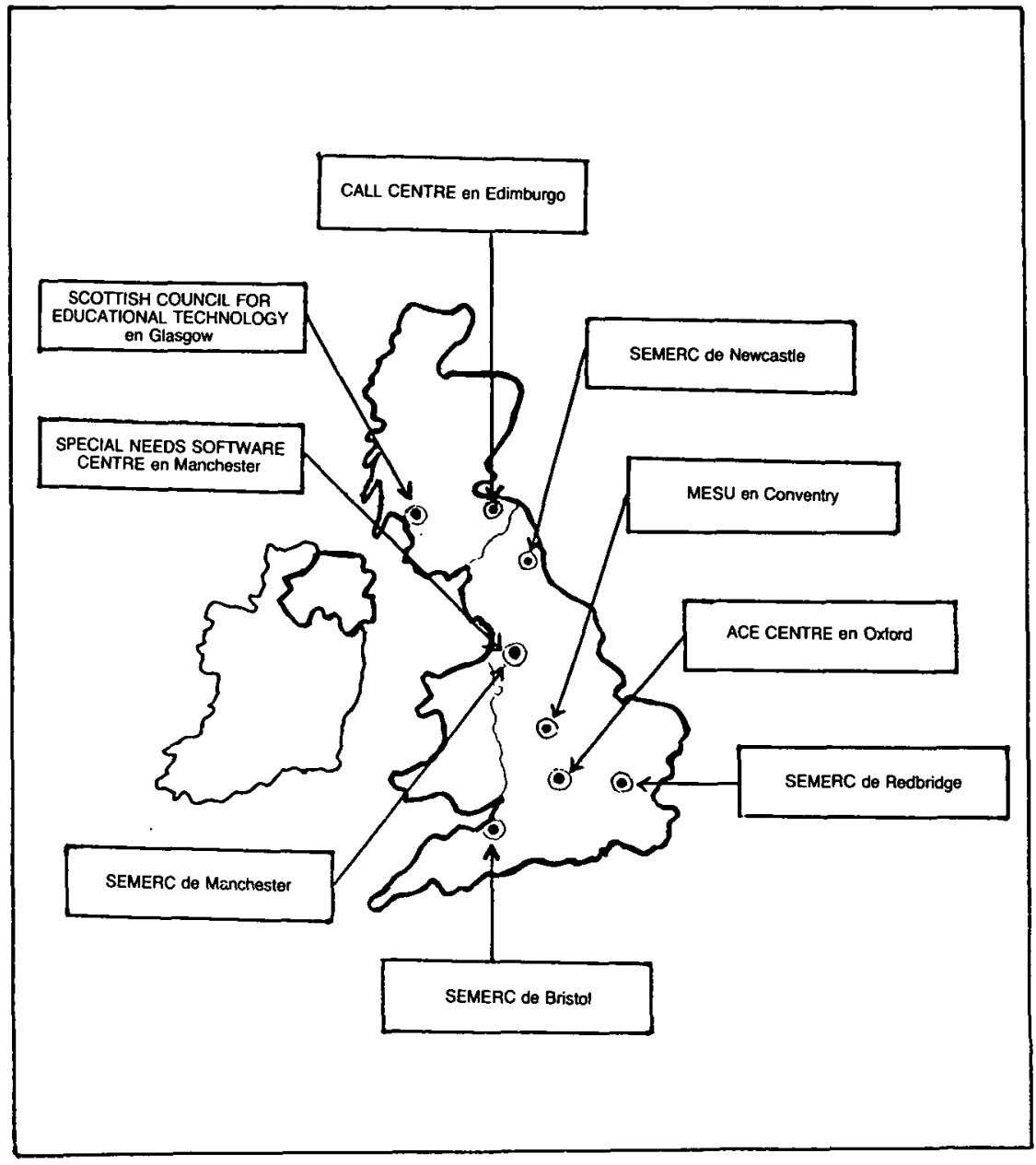

Distribuciön geográfica de los distintos recursos.

FiguRA 2

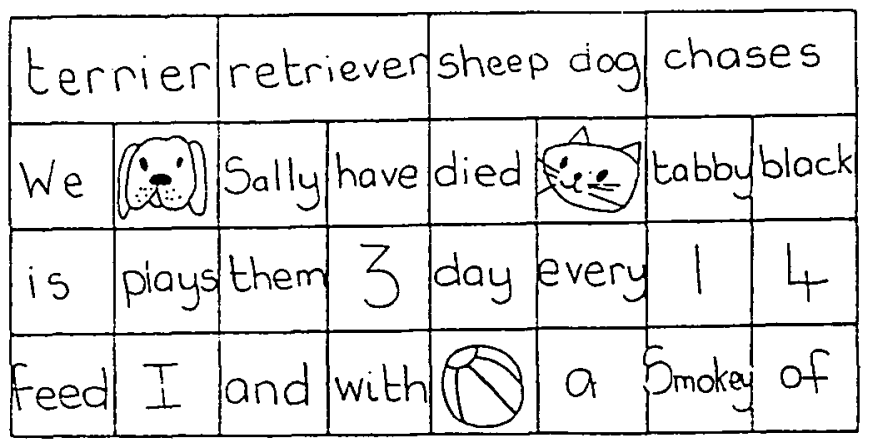

- Overlay* o lámina para teclado de conceptos a partir del vocabulario de una niña galesa con dificultades de lenguaje. Ejemplo de utilización "ad boc" para un caso concreto. 


\section{Microelectrónica y educación especial en el Reino Unido. J. González. \\ CLE, 1991, 10, 57-69.}

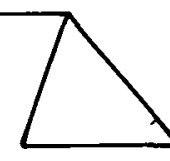

\section{Resumen}

El campo de las aplicaciones de la informática y microelectrónica a la educación especial ha experimentado en los últimos años un notable avance, conquistándose de forma progresiva mayores cotas de comunicación y control del entorno por parte de los niños y niñas discapacitados.

Se presenta aquí una panorámica general al respecto en uno de los paises que con más *tradición* cuentan en este terreno.

Datos sobre el autor: Julio González es psicólogo y está particularmente interesado en la aplicación de la Informática al campo de la psicopedagogía. Actualmente es el director del Servicio Psicopedagógico Escolar de Torrent (Valencia), dependiente de la Consellería de Cultura, Educación y Ciencia de la Generalitat Valenciana.

Dirección: Servicio Psicopedagógico Escolar. Virgen del Olivar 40, 1A. 46900 Torrent (Valencia)

(C) De todos los artículos. Deberá solicitarse por escrito autorización de CLE para el uso en forma de facsímil, fotocopia o cualquier otro medio de reproducción. CLE se reserva el derecho de interponer las acciones legales necesarias en aquellos casos en que se contravenga la ley de derechos de autor. 